\title{
The Combination of Metformin and Valproic Acid Has a Greater Anti-tumoral Effect on Prostate Cancer Growth In Vivo than Either Drug Alone
}

\author{
LINH N.K. TRAN ${ }^{1,2}$, GANESSAN KICHENADASSE $^{1}$, KATHERINE L. MOREL ${ }^{1}$, TINA C. LAVRANOS ${ }^{3}$, \\ SONJA KLEBE ${ }^{4}$, KAREN M. LOWER ${ }^{5}$, REBECCA J. ORMSBY ${ }^{1}$, DAVID J. ELLIOT ${ }^{6}$ and PAMELA J. SYKES ${ }^{1}$ \\ ${ }^{1}$ Flinders Centre for Innovation in Cancer, Flinders University and Medical Centre, Adelaide, South Australia; \\ ${ }^{2}$ University of Medicine and Pharmacy at Ho Chi Minh City, Ho Chi Minh, Vietnam; \\ ${ }^{3}$ Bionomics Ltd, Thebarton, South Australia; \\ ${ }^{4}$ Department of Anatomical Pathology, Flinders University and Medical Centre, Adelaide, South Australia; \\ ${ }^{5}$ Molecular Medicine and Pathology, Flinders University and Medical Centre, Adelaide, South Australia; \\ ${ }^{6}$ Clinical Pharmacology, Flinders University and Medical Centre, Bedford Park, Adelaide, South Australia
}

\begin{abstract}
Background/Aim: The hypoglycemic drug metformin (MET) and the anti-epileptic drug valproic acid (VPA) have individually shown anti-tumor effects in prostate cancer in vitro. The present study intended to investigate the efficacy of the combination of MET and VPA in prostate cancer treatment in a pre-clinical xenograft model. Materials and Methods: Prostate cancer cell lines ( $L N C A P$ and $P C-3)$ were inoculated under the skin of BALB/c nude mice. The mice were treated with $200 \mu \mathrm{l} / \mathrm{ml}$ MET and/or $0.4 \%(w / v)$ VPA diluted in drinking water, or with vehicle control, and were monitored until the tumor volume reached 2,000 $\mathrm{mm}^{3}$. Evaluation of toxicity of the drug combination was determined in liver and kidney by histology. Results: In both LNCaP and PC-3 xenografts, MET combined with VPA significantly reduced tumor growth during the first 4 weeks following treatment, and delayed the time-to-tumor volume of 2,000 $\mathrm{mm}^{3}$ by 90 days, as compared to MET or to VPA alone, and to vehicle control. There was no significant difference in total mouse weight, liver or kidney morphology in response to combination treatment (MET+VPA) compared to MET or VPA alone and vehicle control. Conclusion. The combination treatment of MET with VPA is more effective at
\end{abstract}

This article is freely accessible online.

Correspondence to: Linh N.K. Tran, University of Medicine and Pharmacy at Ho Chi Minh City, Ho Chi Minh City, Vietnam; Flinders Centre for Innovation in Cancer, Flinders University and Medical Centre, Bedford Park, Adelaide, South Australia. Tel: +84 989047088, e-mail: khaclinh@gmail.com, linh.tran@ flinders.edu.au, khaclinh@ump.edu.vn

Key Words: Metformin, valproic acid, prostate cancer chemotherapy, xenograft, nude mice. slowing prostate tumor growth in vivo compared to either drug alone, in mouse xenografts. These pre-clinical results support previous in vitro data and also demonstrate the low toxicity of the combination of these drugs, suggesting that this may be a potential new therapy to be investigated in clinical trials for prostate cancer.

Prostate cancer $(\mathrm{PCa})$ represents the highest incidence of cancer among male patients in the Western world (1-3). Early stages of the disease can be treated with active surveillance, radical prostatectomy, and radiation therapy (external-beam radiation or low dose-rate brachytherapy), while the advanced or relapsing disease needs either hormonal therapy or chemotherapy $(4,5)$. The management of patients that develop castrate-resistant prostate cancer (CRPC) following hormonal therapy remains a challenge with a low median survival time of 12-15 months (6). Current chemotherapies only extend the survival time of CRPC by a mean of 3-6 months (7-10) and are associated with significant cytotoxic effects $(9,11)$. Therefore, it is clear that new cancer therapies are required for more robust cancer control, preferably with minimal toxicity.

Metformin (MET) is the first-line therapy for controlling glucose levels in blood and has been widely used in diabetic patients (12). The pleiotropic beneficial effects of MET have been evident in the polycystic ovarian syndrome (13), in nonalcoholic fatty liver disease (14), in premature puberty (15), as well as in cancer prevention $(16,17)$. Inhibition of the mammalian target of rapamycin (mTOR) (18-21) and of cyclin D1 contributes to the anti-cancer effect of MET (18). A recent epidemiological study has demonstrated that overall $\mathrm{PCa}$ risk decreases in diabetic patients treated with MET $(\mathrm{HR}=1.44,95 \% \mathrm{CI}=1.09-1.91)(22)$, while a single-arm phase 
II clinical trial has found that MET can delay the progression of metastatic $\mathrm{PCa}$, as measured by prostate-specific antigen (PSA) doubling time (23). However important these findings may be, they have been obtained after only a short period of treatment with no control arm included in the studies.

Valproic acid (VPA) is mainly indicated for epilepsy treatment (24). VPA has been shown to have potential as an anti-cancer therapeutic drug through its broad range activity as a histone deacetylase (HDAC) inhibitor targeting HDAC class I and II (IIa) enzymes $(25,26)$. The antineoplastic effects of VPA may be attributed to its regulation of cellular activities that are important in cancer cell growth including cell cycle control, cell differentiation, DNA repair, and apoptosis (27-30). However, the only phase II clinical trial of VPA for prostate cancer failed to achieve the optimal pharmacological level due to its toxicity (31).

Combination therapy targeting multiple neoplastic pathways is likely to be more effective than monotherapy (32). Both MET and VPA appear promising as anti-cancer agents, but at doses required for anti-cancer effects they both exhibit limitations related to toxicity $(31,33)$. However, MET and VPA act via different molecular biological pathways even though they both induce cell-cycle arrest, and have anti-proliferative and anti-apoptotic effects $(18,25)$. Under this scope, we hypothesized that the combination of MET with VPA may be more effective as an anti-cancer therapy than either drug alone, potentially allowing for lower drug doses to be effective in the combination treatment.

We recently reported that MET and VPA in combination synergistically reduced the proliferation of prostate cancer cell lines in vitro (PC-3 and LNCaP), with minimal adverse effects in normal prostatic epithelial cells (34). This combination also synergistically induced cell apoptosis in the presence of p53 and the androgen signaling pathway (34). An additional in vitro report has demonstrated that MET combined with VPA act synergistically as anti-proliferative and pro-apoptotic agents in two clear cell renal cell carcinoma cell lines, but no mechanistic data were included in this study (35). There is, however, in vivo evidence showing that treatment exclusively with MET or VPA alone delays the growth of prostate cancer xenografts $(18,36)$. Here, we demonstrate that the combination of MET and VPA at doses that do not cause any obvious liver or kidney damage, induce a greater anti-tumor effect compared to MET or VPA alone in prostate cancer cell line xenografts. These results suggest that chronic administration of MET combined with VPA may provide an effective low toxicity therapy for prostate cancer patients.

\section{Materials and Methods}

In vivo experimental design. The overall experimental design is shown in Figure 1 for LNCaP and PC-3 cells. This study was approved by the Flinders University Animal Welfare Committee (AWC Approval 893/15). Briefly, female nude (nu/nu) mice were obtained at 5 weeks of age and allowed to acclimate in the animal facility for one week. The mice were injected with the appropriate number of LNCaP or PC-3 cells determined in pilot studies (unpublished data) and the tumors allowed to grow to a volume of $100 \mathrm{~mm}^{3}$. The mice were then randomized into 4 different groups ( $n=10$ /group), which received one of the following in normal drinking water for a total period of 8 weeks: i) drinking water; ii) $200 \mu \mathrm{g} / \mathrm{ml}$ MET; iii) $0.4 \%$ (w/v) VPA in water; iv) $200 \mu \mathrm{g} / \mathrm{ml}$ MET $+0.4 \%$ (w/v) VPA. When the tumors reached a volume of $2,000 \mathrm{~mm}^{3}$, peripheral blood was collected from the submandibular vein to assess plasma levels of MET and VPA, and then the mice were euthanized and the tumors were harvested for further analysis.

In the PC-3 xenograft group, one mouse was euthanized on day 2 (MET+VPA group) and one on day 3 (VPA group) of treatment, due to lesions sustained from fighting, while one mouse in the VPA group was euthanized at day 14 due to chylothorax (cause unknown). One mouse with LNCaP xenograft in the MET+VPA treatment group was euthanized at day 32 due to a tumor-associated motility problem, and one mouse with a PC-3 xenograft in the MET+VPA treatment group was euthanized due to tumor ulceration at day 58. These mice were not included in our analyses. The experimental design and final numbers of animals analyzed are summarized in Figure 1.

Cell culture and chemicals. LNCaP (clone FGC) (CRL-1730) and PC-3 (CRL-1435) prostate cancer cell lines were newly obtained from the American Type Culture Collection $\left(\right.$ ATCC $^{\circledR}$, Manassas, Virginia, USA). Metformin (PHR1084-500MG) and valproic acid (P4543-10G) were purchased from Sigma-Aldrich (Castle Hill, New South Wales, AU) and stocks (15 mg/ml for each drug) were made in distilled water and were sterilized using a $0.22 \mu \mathrm{m}$ filter. Cancer cell lines were grown in RPMI 1640 medium (ThermoFisher, Melbourne, Victoria, AU) supplemented with $100 \mathrm{U} / \mathrm{ml}$ penicillin, $100 \mu \mathrm{g} / \mathrm{ml}$ streptomycin and $5 \%$ fetal bovine serum (FBS; Bovogen, Melbourne, Victoria, AU). When cells reached $80-90 \%$ confluence, they were harvested using $0.05 \%$ Trypsin/0.48 mM EDTA (ThermoFisher).

Establishment of tumor xenografts. Balb/c nude male mice (BALB/c-Fox1 $\left.{ }^{n u} / A r c\right)$ were obtained from the Animal Resources Centre (Perth, Australia). LNCaP and PC-3 were grown up to 80$90 \%$ confluence and were harvested on the day of inoculation. The cells were washed briefly with PBS and were then centrifuged at $160 \times g$ for $5 \mathrm{~min}$ at room temperature. Cell pellets were resuspended in $50 \mu \mathrm{l}$ PBS and the same volume of Corning ${ }^{\circledR}$ Matrigel ${ }^{\circledR}$ Matrix (In Vitro Technologies, Melbourne, Victoria, AU) was added to the cell suspension to obtain $1 \times 10^{6}$ cells for the PC3 and $3 \times 10^{6}$ cells for the $\mathrm{LNCaP}$ line in a final volume of $100 \mu \mathrm{l}$ at $4^{\circ} \mathrm{C}$. Cell suspensions were kept on ice until they were injected subcutaneously into the right hind flank. All animals were subsequently checked on a daily basis for their general health condition, including faecal consistency, evidence of dehydration, general movement and breathing. Mouse weight and tumor size were measured 3 times per week throughout the study. Tumor volume was calculated using the formula $(\pi / 6) \times \mathrm{A} \times \mathrm{B} 2$ where $\mathrm{A}$ was the larger tumor diameter and $\mathrm{B}$ was the smaller tumor diameter $(18,37)$. When the tumor reached a volume of 2,000 $\mathrm{mm}^{3}$, the mice were euthanized and the tumor, liver and kidneys were harvested. Gross necropsy of the animals was performed to ensure that there were no confounding health conditions. 


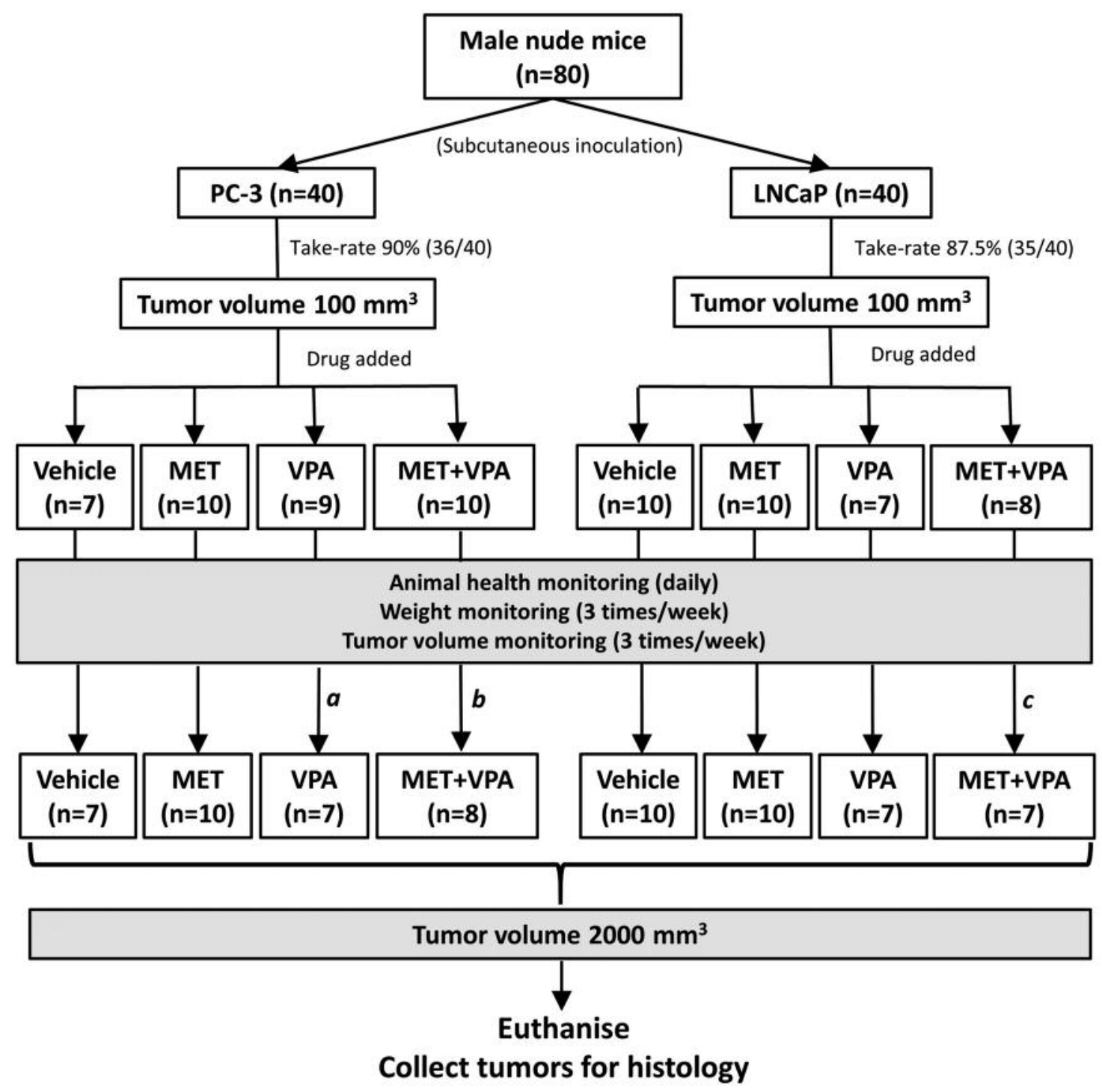

Figure 1. Study design summary of metformin (MET) plus valproic acid (VPA) treatment in mice with PC-3 or LNCaP xenografts. Six-week-old nude mice $(n=80)$ were randomly assigned into two groups and inoculated with either LNCaP $(n=40)$ or PC-3 (n=40) cells. Once the xenograft tumor reached $100 \mathrm{~mm}^{3}$, mice were randomly assigned into one of 4 groups ( $n=10 /$ group), treated with $i$ ) vehicle control, ii) $200 \mu \mathrm{g} / \mathrm{ml} \mathrm{MET}$ alone, iii) $0.4 \%(w / v)$ VPA alone, and iv) $200 \mu \mathrm{g} / \mathrm{ml}$ MET $+0.4 \%(w / v)$ VPA. The xenograft tumors were allowed to grow until a maximum volume of

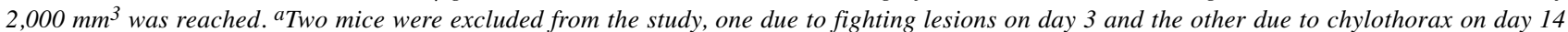
of treatment. ${ }^{b}$ One mouse was excluded from the study due to fighting lesions on day 2 and one mouse was censored on day 58 of treatment due to tumor ulceration. ${ }^{c}$ One mouse was censored on day 32 of treatment due to tumor affecting mouse movement.

\begin{abstract}
Administration of MET and VPA. MET and VPA were added to the drinking water and this was replaced with a fresh solution every 3.5 days $(36,38)$. The drug concentrations of MET at $200 \mu \mathrm{g} / \mathrm{ml}$ and VPA at $0.4 \%(\mathrm{w} / \mathrm{v})$ in water were based on previous studies where MET and VPA on their own were shown to reduce LNCaP xenograft growth $(18,36)$.
\end{abstract}

Plasma concentrations of MET and VPA using mass spectrometry. An initial pilot study was performed ( $\mathrm{n}=3$ for each treatment group) to verify the presence of MET and VPA in peripheral blood plasma using ultra-performance liquid chromatography-mass spectrometry (UPLC-MS). The protocols were adapted for mice. Peripheral blood (50-100 $\mu \mathrm{l})$ was collected from the submandibular vein of mice. The plasma was isolated from total blood by centrifuging at $1,000 \times g$ for $10 \mathrm{~min}$ at $4^{\circ} \mathrm{C}$, which was then stored at $-20^{\circ} \mathrm{C}$ for subsequent analysis. Plasma samples $(20 \mu \mathrm{l})$ for MET detection were injected onto a Phenomenex Kinetex HILIC column $(2.1 \times 100 \mathrm{~mm}, 2.6 \mu \mathrm{m})$ and were analyzed using an AcquityTM Ultra Performance LC (Waters, Rydalmere, New South Wales, AU). For VPA detection, UPLC-MS analysis was performed using an AcquityTM Ultra 
Performance LC system (Waters) coupled to a Premier qToF mass spectrometer (Waters). The mass spectrometer was operated on negative ionisation mode with a capillary voltage at $2.6 \mathrm{kV}$, a source temperature at $100^{\circ} \mathrm{C}$, a desolvation temperature $300^{\circ} \mathrm{C}$, a sample cone voltage of $26 \mathrm{~V}$ and a collision energy of $6 \mathrm{~V}$. VPA was detected on tandem mass spectrometry mode by pseudo multiple reaction monitoring at the parent ion mass $[\mathrm{M}-\mathrm{H}]-=143.1 \mathrm{Da}$.

Evaluation of liver and kidney histopathology. Liver and kidney tissues were fixed in $10 \%$ formalin (Sigma-Aldrich) overnight, and were then processed using an STP 120 Spin Tissue Processor (ThermoFisher). The processed tissues were embedded in paraffin (\#Paraplast ${ }^{\circledR}$, Surgipath ${ }^{\circledR}$, Melbourne, Victoria, AU) using a Histostar $^{\mathrm{TM}}$ Embedding Workstation (ThemoFisher). Hematoxylin (\#II500JJ, ThermoFisher) and Eosin (\#HT110116, Sigma-Aldrich) (H\&E) staining was performed using the routine diagnostic protocol of the Pathology Department, Flinders Medical Centre, Adelaide, AU. In brief, paraffin embedded tissues were cut into 5 - $\mu$ m sections using a Microtome (Leica, Melbourne, Victoria, AU). The sections were mounted on APES (3-aminopropyltriethoxysilane) coated slides. The remaining paraffin was removed by placing the slides in an oven at $70^{\circ} \mathrm{C}$ for $20 \mathrm{~min}$, and washing the slides twice with the Histochoice ${ }^{\circledR}$ Clearing Agent (\#H2779, Sigma-Aldrich) for 2 min with shaking. The sections were rehydrated by washing twice each in $100 \%, 95 \%, 70 \%$ and $50 \%$ ethanol for 1 min per wash with shaking, followed by a final wash in tap water. The slides were quickly dipped in acid ethanol and ammonia water before staining with Eosin for $2 \mathrm{~min}$ followed by Haematoxylin stain for $2 \mathrm{~min}$. The slides were rehydrated serially in tap water, ethanol, and Histochoice ${ }^{\circledR}$ Clearing Agent, allowed to dry, and then mounted with Leica CV Mount (Leica, Melbourne, Victoria, AU) and imaged using an Olympus microscope (BX63). The histological score adapted from Chen et al. (39), was used to evaluate the glomerular changes (hypercellularity, hypertrophy, cellular crescent formation, thrombotic changes, fibrinoid material deposition), tubular dilation, necrosis, and inflammation. The toxicity of the liver was investigated using the histological score adapted from Mendler et al. (40) to evaluate portal fibrosis, lobular inflammation or necrosis, Mallory bodies, hepatocyte ballooning, peri-sinusoidal fibrosis, and indications of fatty liver diseases.

Statistical analysis. IBM SPSS 23 was used for statistical analyses. Differences in tumor volumes in vivo were analyzed using a twoway ANOVA test. Kaplan-Meier analysis was used to plot the timeto-tumor volume of up to $2,000 \mathrm{~mm}^{3}$ in the different treatment models.

\section{Results}

Chronic administration of MET combined with VPA in drinking water does not cause significant adverse effects. A pilot study was conducted to investigate possible adverse effects in nude mice in the absence of tumor xenografts in response to an 8-week treatment with either vehicle, $200 \mu \mathrm{g} / \mathrm{ml} \mathrm{MET} \mathrm{alone,} 0.4 \%(\mathrm{w} / \mathrm{v})$ VPA alone, or $200 \mu \mathrm{g} / \mathrm{ml}$ MET $+0.4 \%(\mathrm{w} / \mathrm{v})$ VPA treatment $(\mathrm{n}=3$ in each group). The mice exhibited no side-effects as a result of any treatment. There was no significant variation in mouse weight observed between the vehicle, MET alone, VPA alone, and combination treatment (MET+VPA) groups throughout the course of experiment $(p>0.05)$ (Figure 2A). Histological scoring of the kidney (Figure 2B) and liver (Figure 2C) showed no damage $($ score $=0)$ to either organ in any of the treatment groups.

Drinking consumption of MET and VPA in the nude mice was evaluated. The presence of MET and/or VPA in peripheral blood plasma was confirmed in all animals at 4 and at 8 weeks of treatment. As the drinking water containing the drugs was provided ad libitum, and the assays were semi-quantitative, it was not possible to accurately determine the exact drug concentrations in the blood.

MET combined with VPA causes greater reduction of tumor growth in both PC-3 and LNCaP xenografts than either drug alone. In order to investigate the effect of MET and VPA in tumor growth of PC-3 and LNCaP xenografts, 6-week-old male mice were inoculated with $\operatorname{LNCaP}(n=40)$ or PC-3 $(n=40)$, then they were randomly assigned into vehicle control (water), MET, VPA, or MET+VPA treatment groups $(n=10 /$ treatment group/cell line). Once the xenograft tumor reached $100 \mathrm{~mm}^{3}$, mice were treated with either the vehicle control, $200 \mu \mathrm{g} / \mathrm{ml}$ MET alone, $0.4 \%$ (w/v) VPA alone, or $200 \mu \mathrm{g} / \mathrm{ml} \mathrm{MET}+0.4 \%(\mathrm{w} / \mathrm{v}) \mathrm{VPA}$. There were no significant differences in the time taken for the tumors to reach $100 \mathrm{~mm}^{3}$ in any of the four treatment groups and for either cell line. The tumor-take rate in this study was $87.5 \%$ (35/40) for LNCaP and 90\% (36/40) for PC-3 cells. No spontaneous tumor regression was observed in the study. Tumor volumes were compared after each treatment was added, using the cut-off time of when the first PC-3 or LNCaP xenograft tumor reached $2,000 \mathrm{~mm}^{3}$, which was 28 days for PC-3 and 21 days for LNCaP xenografts. Mice euthanized before the cut-off time (one mouse in the VPA and one in the MET+VPA group both with PC-3 xenografts and one mouse in the VPA group with LNCaP xenografts) were excluded from analysis and mice euthanized after the cut-off time were removed from the Kaplan-Meier analysis (one mouse in the MET+VPA group with PC-3 and one in the same group with LNCaP xenografts).

In the PC-3 xenograft group, there was no significant difference in tumor volume in mice treated with MET alone, VPA alone, or with vehicle alone $(p>0.05)$. The combination of MET with VPA significantly inhibited the volume of the PC-3 tumor xenograft $(86.7 \%$ decrease, $p=0.001)$, whereas the MET alone $(63.9 \%$ decrease, $p=0.005)$, and VPA alone (59.7\% decrease, $p=0.04$ ) (Figure $3 \mathrm{~A}$ and $\mathrm{C}$ ) had a less robust effect, as compared to the vehicle alone model on day 28 of the treatment.

In the LNCaP xenograft group, MET alone inhibited tumor growth compared to vehicle treatment $(40.1 \%$ decrease, $p<0.001)$, as did VPA alone (42.2\% decrease, $p<0.001$ ) (Figure 3B and D). MET combined with VPA 

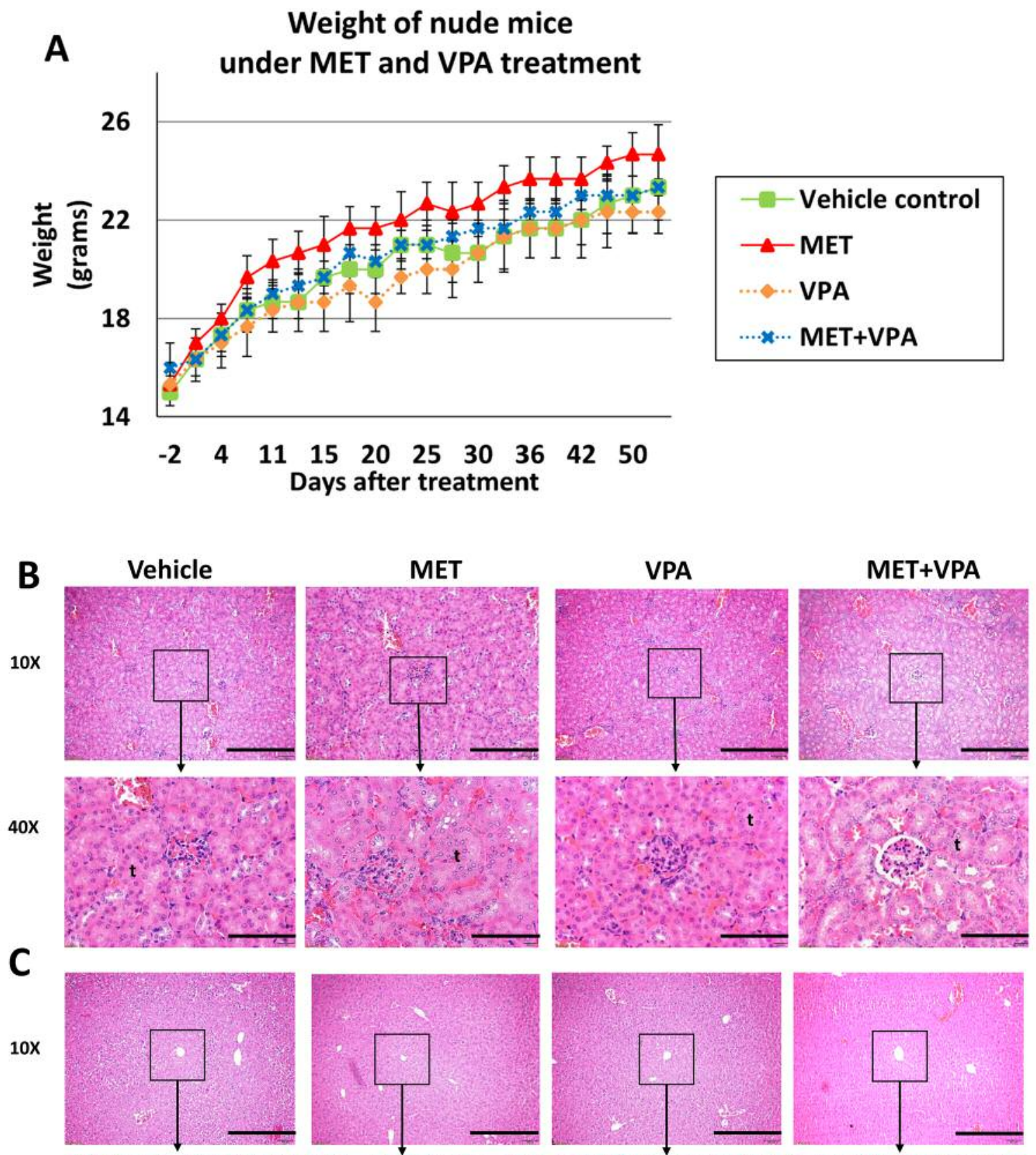

$40 X$
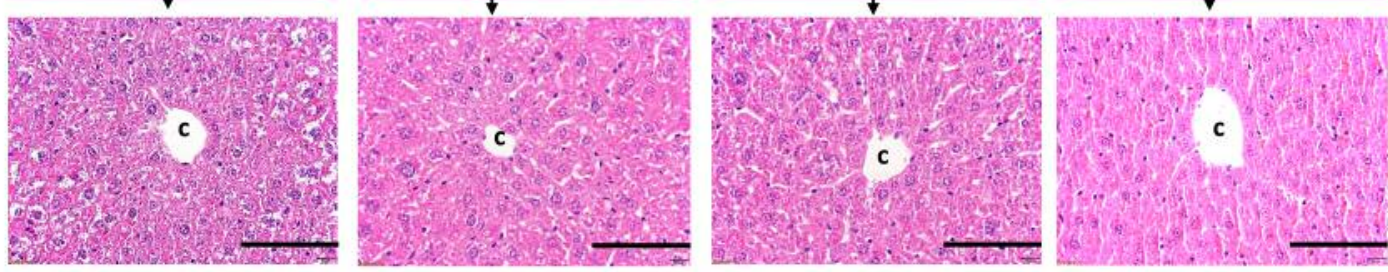

Figure 2. The weight of nude mice (mean \pm SE) was evaluated before and after treatment with MET alone, VPA alone, MET plus VPA, or vehicle control $(n=3)$. There was no significant difference in weight between the groups over 8 weeks of treatment ( $p>0.05)(A)$. Representative hematoxylin and eosin staining images (10x and 40x) of the kidney $(B)$ and the liver $(C)$, during week 8 of treatment. The histological scoring of the kidney $(B)$ showed no tubule $(t)$ changes, thrombosis, dilation, necrosis, or inflammation (score 0 ). The histological scoring of the liver $(C)$ showed no signs of portal fibrosis around central vein (c), lobular inflammation or necrosis, Mallory bodies, hepatocyte ballooning, perisinusoidal fibrosis, or fatty changes (score 0). Scale bar $4 \mathrm{~mm}$ for 10x images, and $80 \mu \mathrm{m}$ for $40 \times$ images. 
A

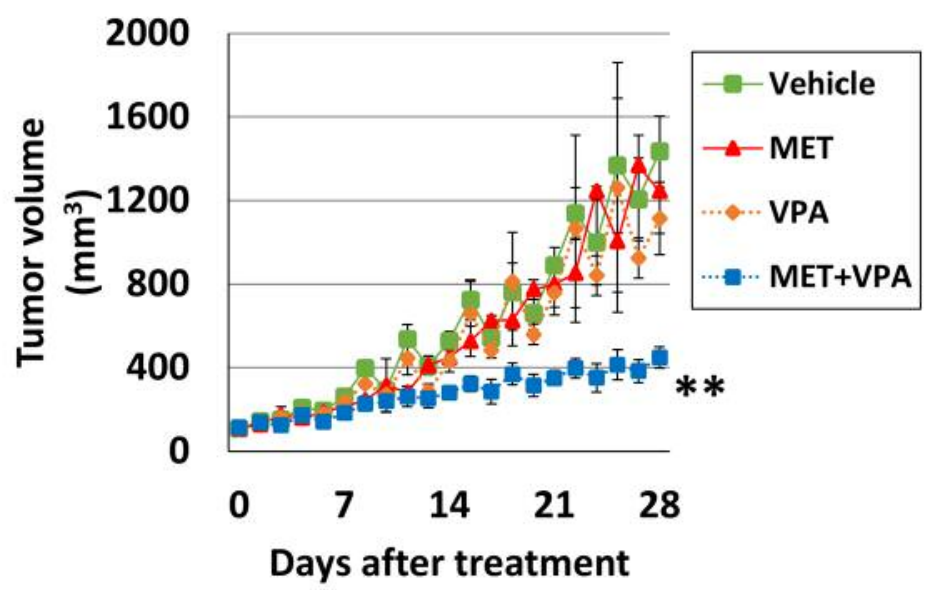

B

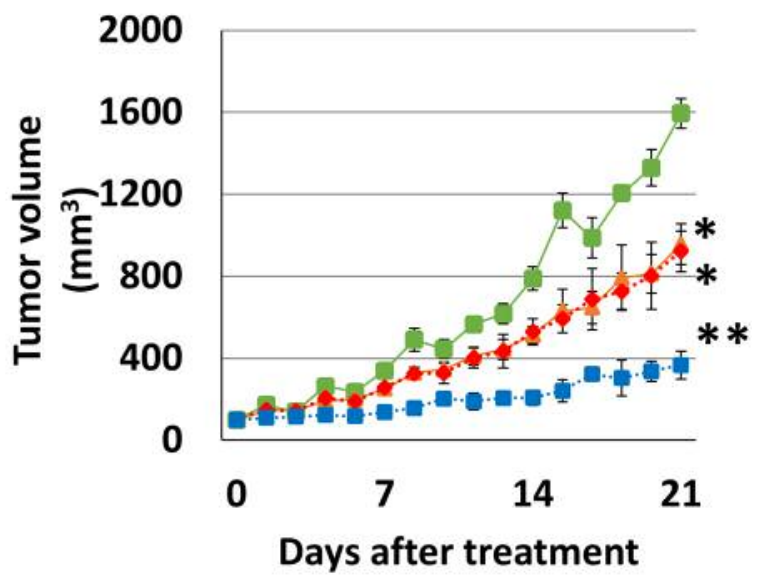

C

\begin{tabular}{|l|c|c|c|}
\hline Treatment & N & Mean volume & SE \\
\hline Vehicle & 7 & 1434.5 & 169.6 \\
\hline MET & 9 & 1244.2 & 201.6 \\
\hline VPA & 7 & 1114.5 & 173.4 \\
\hline MET+VPA & 9 & 449.4 & 51.0 \\
\hline
\end{tabular}

D

\begin{tabular}{|l|c|c|c|}
\hline Treatment & $\mathbf{N}$ & Mean volume & SE \\
\hline Vehicle & 10 & 1594.7 & 72.1 \\
\hline MET & 10 & 955.5 & 99.2 \\
\hline VPA & 7 & 921.7 & 99.5 \\
\hline MET+VPA & 8 & 366.7 & 68.3 \\
\hline
\end{tabular}

Figure 3. Tumor growth in response to metformin (MET) and/or valproic acid (VPA) treatment. Nude mice at 6 weeks of age were inoculated subcutaneously with PC-3 (A) or LNCaP (B) cells. The xenograft tumors were allowed to grow to $100 \mathrm{~mm}^{3}$ before treating the mice with either vehicle control, $\left.200 \mu \mathrm{g} / \mathrm{ml} \mathrm{MET} \mathrm{alone,0.4 \%} \mathrm{(w/v)} \mathrm{VPA} \mathrm{alone,} \mathrm{or} 200 \mu \mathrm{g} / \mathrm{ml} \mathrm{MET+0.4 \%} \mathrm{(w/v)} \mathrm{VPA.} \mathrm{The} \mathrm{sample} \mathrm{size} \mathrm{(N),} \mathrm{mean} \mathrm{tumor} \mathrm{volume} \mathrm{(mm}{ }^{3}\right)$ and standard error (SE) of PC-3 xenografts on day 28 of treatment and LNCaP xenografts on day 21 of treatment are presented in tables C and D, respectively. ${ }^{*} p<0.05$ compared to the vehicle, MET alone, and VPA alone. **p<0.005 compared to the vehicle, MET alone, VPA alone, and $M E T+V P A(7 \leq n \leq 10)$.

significantly inhibited tumor growth $(77 \%$ decrease, $p<0.001)$, with less dramatic changes in the MET alone $(61.6 \%$ decrease, $p<0.001)$, and the VPA alone $(60.2 \%$ decrease, $p<0.001)$ treatment, as compared to the vehicle alone model on day 21 of the treatment (Figure $3 \mathrm{~B}$ and D).

Chronic administration of MET combined with VPA delays time-to-maximum tumor volume in LNCAP and PC-3 xenografts treated with MET and VPA. Throughout this study, there was no tumor regression observed in the absence of treatment. Mice were euthanized when the tumor volume reached 2,000 $\mathrm{mm}^{3}$. Most of the tumor xenografts reached $2,000 \mathrm{~mm}^{3}$ before day 90 following drug treatment initiation, with the exception of one LNCaP xenograft tumor which decreased to an undetectable size in the MET plus VPA group and one PC-3 xenograft in the MET plus VPA group which only reached $350 \mathrm{~mm}^{3}$. These mice were culled on day 102 and 114, respectively, as they exhibited no changes in tumor size for 2 weeks prior to euthanasia.

Analysis of cumulative survival using the Kaplan-Meier method showed that MET combined with VPA significantly delayed the time-to-maximum tumor volume in PC-3 xenografts compared to vehicle treatment $(92.2 \%$ increase, $p<0.001)$, MET alone ( $60.5 \%$ increase, $p=0.002)$, and VPA alone $(43.7 \%$ increase, $p=0.01)$. Treatment of nude mice bearing PC 3 xenografts with VPA alone also delayed the time-to-maximum tumor volume compared to the vehicle treatment $(32.8 \%$ increase, $p=0.006)$, whereas the difference was not significant for the MET alone compared to the vehicle treatment group ( $p=0.08)$ (Figure 4A).

In the LNCaP xenograft group, the time-to-maximum tumor volume in response to MET plus VPA was significantly delayed compared to MET alone (44.2\% increase, $p=0.002$ ), VPA alone (43.9\% increase, $p=0.001)$, and vehicle treatment $(120 \%$ 

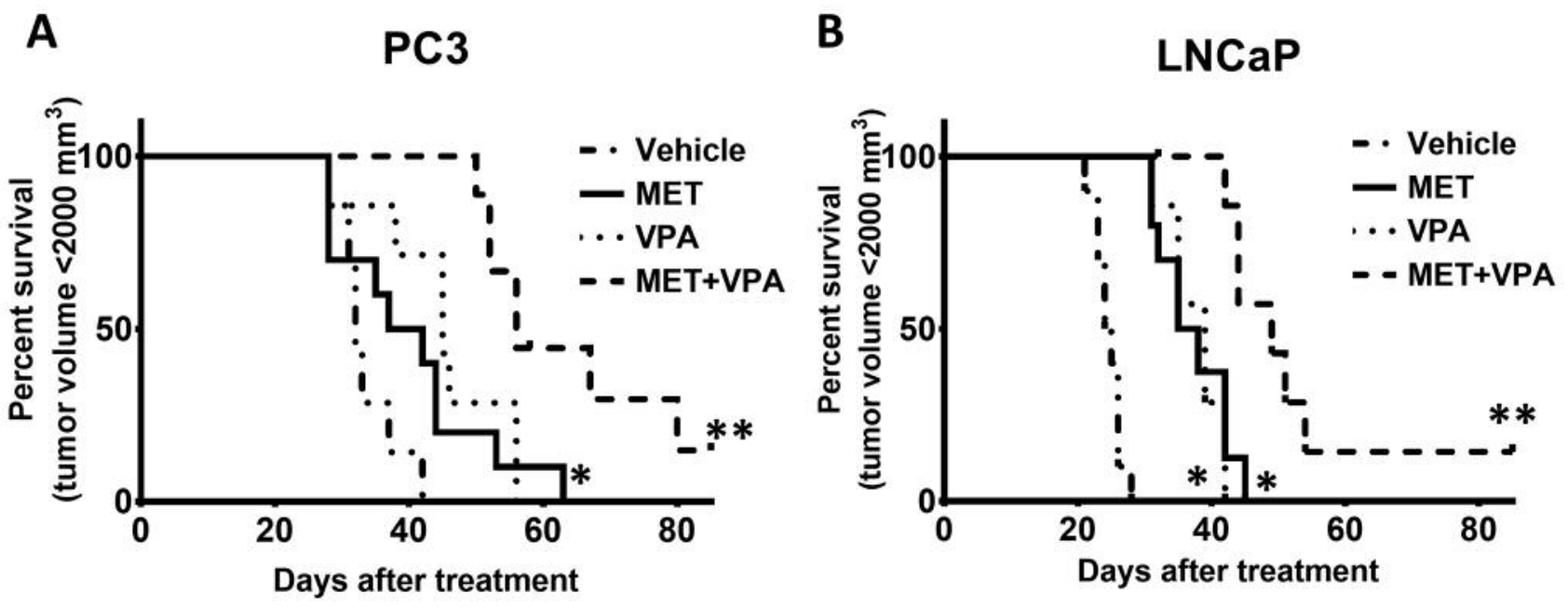

Figure 4. Kaplan-Meier analysis of percentage of animals reaching a tumor volume of 2,000 $\mathrm{mm}^{3}$ during the treatment with metformin (MET) and valproic acid (VPA). Mice bearing tumor xenografts with either PC-3 or LNCaP were treated with vehicle control, MET alone, VPA alone, or MET plus VPA. The mice were euthanized when the tumor volume reached 2,000 $\mathrm{mm}^{3}$. One mouse bearing a PC-3 xenograft in the MET+VPA treatment group only reached a tumor size of $350 \mathrm{~mm}^{3}$ and was euthanized on day 114 of treatment, while the tumor of one mouse bearing a LNCaP xenograft in the MET+VPA treatment group was reduced to an undetectable size and was euthanized on day 102 of treatment. *p<0.05 compared to vehicle treatment; $* * p<0.005$ compared to vehicle treatment, MET alone, and VPA alone $(7 \leq n \leq 10)$.

increase, $p<0.001)$. Treatment with either MET or VPA alone significantly delayed the time-to-tumor volume of $2,000 \mathrm{~mm}^{3}$ compared to vehicle treatment $(52.4 \%$ increase, $p<0.001$ and $52.8 \%$ increase, $p<0.001$, respectively) (Figure 4B).

On necropsy, only one abdominal lymph node metastasis was identified in a mouse with a PC-3 xenograft (1/36) in the vehicle treatment group (day 33 after treatment), while no metastases of LNCaP xenografts $(0 / 35)$ were observed.

\section{Discussion}

Our previous in vitro findings have demonstrated that the combination treatment of MET and VPA induces a synergistic anti-proliferative response in $\mathrm{LNCaP}$ and $\mathrm{PC}-3$ cell lines with no significant side-effects in normal prostatic epithelial cells (PrEC) (34). A significant synergistic apoptotic response was observed in vitro in $\mathrm{LNCaP}$, but not in PC-3 cells. MET combined with VPA reduced proliferation and induced apoptosis in human prostate tumor biopsy explants (34). The presence of p53 and the androgen signaling pathway were shown to play an important role in the synergistic apoptosis of prostate cancer cell lines in response to MET+VPA $(34,41)$. Here, we performed an in vivo study to investigate the combination of MET and VPA in PC-3 and LNCaP xenografts in nude mice to support these in vitro findings and provide preliminary safety evidence for the use of this drug combination in clinical studies. In our xenograft experiments $200 \mu \mathrm{g} / \mathrm{ml}$ of MET and $0.4 \%$ (w/v) of VPA were diluted in drinking water, which relates to human equivalent doses of 2.0-4.1 mg/kg/day for MET $(42,43)$ and 40.6-79.4 mg/kg/day for VPA (36). These doses are in the therapeutic range and are already being used for non-cancer purposes in humans with minimal adverse effects (44).

The combination of MET with VPA did not cause any kidney or liver toxicity. In previously published xenograft studies mice were administered MET alone, or VPA alone in drinking water, at the same doses as used here, with no health-related issues $(18,36)$. However, there are currently no reports on tissue toxicity in response to the combination of MET and VPA. The mice in our study showed no general side-effects during the 8 weeks of receiving $200 \mu \mathrm{g} / \mathrm{ml} \mathrm{MET,} \mathrm{0.4 \%} \mathrm{(w/v)}$ VPA or both drugs combined in their drinking water. Gross necropsy indicated no tissue toxicity and histopathological analyses of liver and renal tissue (major metabolic sites of MET and VPA, respectively) showed no evidence of damage. These results suggest that chronic administration of MET and VPA can be combined at the doses used here with possibly minimal side-effects to the liver or kidney.

The combined treatment of MET with VPA inhibits tumor growth. There was no natural tumor regression observed after tumors reached $100 \mathrm{~mm}^{3}$ in our pilot study, therefore drug treatment was commenced at this point. Our current study used Matrigel ${ }^{\circledR}$ for inoculating the cancer cell lines and the tumor take-rate was $87.5 \%$ for the $\mathrm{LNCaP}$ and $90 \%$ for the PC-3 xenograft. These results show a stronger effect than the one reported by Davoodpour et al. (45) who used culture medium 
for inoculating cancer cells in the xenograft, but are comparable to those of Gustavsson et al. (46), who, similarly to us, used Matrigel ${ }^{\circledR}$. In the study presented here, subcutaneous injections of PC-3 and LNCaP cells were performed and a low metastatic rate at $2.8 \%(1 / 36)$ was shown in PC-3 xenografts, while no metastases in LNCaP xenografts $(0 / 35)$, in line with the literature $(36,37,39,40)$. No significant differences in tumor growth of PC-3 xenografts were observed in response to either MET or VPA alone compared to the vehicle treatment over the first 4 weeks of treatment. Our findings differ from the report by Shabbeer et al. (2007), where the administration of $0.4 \%$ $(\mathrm{w} / \mathrm{v})$ VPA in drinking water of nude mice for 4 weeks significantly reduced the PC3 xenograft tumor volume by $57 \%$ compared to the vehicle treatment $(p=0.05, \mathrm{n}=8)(47)$. This inconsistency could be attributed to the different time-point for treatment initiation, which was 2 weeks after inoculation in the study of Shabbeer et al. (2007) versus 41.2 \pm 9.4 days after inoculation (when the tumor reached $100 \mathrm{~mm}^{3}$ ) in our study. Although no anti-tumoral effect of VPA alone was observed at 4 weeks, long-term administration of VPA up to 90 days delayed the tumor progression by $32.8 \%$. Importantly, the combination of MET with VPA, compared to MET alone, VPA alone or vehicle treatment, inhibited the tumor growth of PC-3 xenografts by $59-86 \%$ in both the first 4 weeks of treatment and further extended the time-to-tumor volume of $2,000 \mathrm{~mm}^{3}$ by $43-92 \%$ in long term treatment. There was only one PC-3 xenograft that did not reach a final tumor size of $2,000 \mathrm{~mm}^{3}$ during the study and this was in the MET+VPA treatment group. PC-3 has the characteristics of a small cell neuroendocrine carcinoma, which is highly aggressive and hormone refractory (48). The response of the PC-3 xenografts to MET and VPA combination therapy in our study highlights the potential of this combination treatment as an effective therapy even in castration-resistant prostate cancer.

In LNCaP xenografts, MET or VPA alone reduced the tumor volume by approximately $40 \%$ in the first 4 weeks after treatment and prolonged the time-to-tumor volume of 2000 $\mathrm{mm}^{3}$ by approximately $44 \%$ compared to the vehicle treatment. Our results agree with previous findings, where the addition of MET or VPA alone in the drinking water in nude mice bearing LNCaP xenografts reduced the tumor volume growth after 3-4 weeks of treatment (55\% and $40 \%$ reduction, respectively) $(18,36)$. Combining MET with VPA induced a stronger inhibition of initial tumor growth by $60-77 \%$ in the short-term ( 4 weeks) while the long term treatment further extended the time-to-tumor-volume of $2,000 \mathrm{~mm}^{3}$ by $42-120 \%$ at day 90, indicating the effectiveness of MET plus VPA combination therapy over the MET or VPA monotherapies.

LNCaP cells have normal p53 expression and are responsive to androgen receptor (AR), similar to early-stage localized PCa, while PC-3 cells have no p53 expression and are AR-independent, thus they behave more like CRPC and possibly represent $\mathrm{PCa}$ at different stages (48). We found greater anticancer effects using the combination of MET plus VPA in both initial and long-term treatments in LNCaP xenografts, compared to MET alone, VPA alone or vehicle treatment. There was one LNCaP xenograft in the present study which completely regressed and this was in the MET plus VPA treatment group. These findings suggest that the combination treatment of MET with VPA may be more effective in earlier stages of prostate cancer where p53 expression and AR responsiveness is more common (49).

In conclusion, long-term administration of MET and VPA in combination, at doses lower than the human therapeutic dose of MET for diabetes and at the same human therapeutic dose of VPA for epilepsy, could be a relatively non-toxic and efficient systemic therapy at different stages of prostate cancer. Based on these findings, our group has recently commenced a phase I clinical trial investigating the combination of MET with VPA as a neoadjuvant therapy prior to radical prostatectomy (ACTRN 12616001021460).

\section{Acknowledgements}

This research was supported by a Flinders Medical Centre Foundation Seeding grant, and an Australia Awards PhD scholarship to LNKT (ID ST0009TM3).

\section{References}

1 Australian Institute of Health and Welfare \& Australasian Association of Cancer Registries: Cancer in Australia: An overview 2012. Cancer series 74(CAN 70), 2012.

2 Grönberg H: Prostate cancer epidemiology. Lancet 361(9360): 859-864, 2003.

3 Ferlay J, Shin HR, Bray F, Forman D, Mathers C and Parkin DM: Estimates of worldwide burden of cancer in 2008: Globocan 2008. Int J Cancer 127(12): 2893-2917, 2010.

4 Mottet N, Bellmunt J, Bolla M, Briers E, Cumberbatch MG, De Santis M, Fossati N, Gross T, Henry AM and Joniau S: Eauestro-siog guidelines on prostate cancer. Part 1: Screening, diagnosis, and local treatment with curative intent. Eur Urol 71(4): 618-629, 2017.

5 Cornford P, Bellmunt J, Bolla M, Briers E, De Santis M, Gross T, Henry AM, Joniau S, Lam TB and Mason MD: Eau-estro-siog guidelines on prostate cancer. Part ii: Treatment of relapsing, metastatic, and castration-resistant prostate cancer. Eur Urol 71(4): 630-642, 2017.

6 Denmeade SR and Isaacs JT: Development of prostate cancer treatment: The good news. The Prostate 58(3): 211-224, 2004.

7 Ryan CJ, Smith MR, Fizazi K, Saad F, Mulders PF, Sternberg CN, Miller K, Logothetis CJ, Shore ND and Small EJ: Abiraterone acetate plus prednisone versus placebo plus prednisone in chemotherapy-naive men with metastatic castration-resistant prostate cancer (cou-aa-302): Final overall survival analysis of a randomised, double-blind, placebo-controlled phase 3 study. The Lancet Oncol 16(2): 152-160, 2015.

8 Beer TM, Armstrong AJ, Rathkopf DE, Loriot Y, Sternberg CN, Higano CS, Iversen P, Bhattacharya S, Carles J and Chowdhury S: Enzalutamide in metastatic prostate cancer before chemotherapy. New Engl J Med 371(5): 424-433, 2014. 
9 Tannock IF, de Wit R, Berry WR, Horti J, Pluzanska A, Chi KN Oudard S, Théodore C, James ND and Turesson I: Docetaxel plus prednisone or mitoxantrone plus prednisone for advanced prostate cancer. New Engl J Med 351(15): 1502-1512, 2004.

10 Kantoff PW, Higano CS, Shore ND, Berger ER, Small EJ, Penson DF, Redfern CH, Ferrari AC, Dreicer R and Sims RB: Sipuleucel-T immunotherapy for castration-resistant prostate cancer. New Engl J Med 363(5): 411-422, 2010.

11 Widakowich C, de Castro G, De Azambuja E, Dinh P and Awada A: Review: Side effects of approved molecular targeted therapies in solid cancers. The Oncologist 12(12): 1443-1455, 2007.

12 UK Prospective Diabetes Study Group: Intensive blood-glucose control with sulphonylureas or insulin compared with conventional treatment and risk of complications in patients with type 2 diabetes (ukpds 33). Lancet 352(9131): 837-853, 1998.

13 Lord JM, Flight IH and Norman RJ: Metformin in polycystic ovary syndrome: Systematic review and meta-analysis. BMJ 327(7421): 951-953, 2003.

14 Marchesini G, Bianchi G, Tomassetti S, Zoli M and Melchionda $\mathrm{N}$ : Metformin in non-alcoholic steatohepatitis. Lancet 358(9285): 893-894, 2001.

15 Ibáñez L, Ong K, Valls C, Marcos MV, Dunger DB and de Zegher F: Metformin treatment to prevent early puberty in girls with precocious pubarche. J Clin Endocrinol Metab 91(8): 2888 2891, 2006.

16 Zhou G, Myers R, Li Y, Chen Y, Shen X, Fenyk-Melody J, Wu M, Ventre J, Doebber T and Fujii N: Role of AMP-activated protein kinase in mechanism of metformin action. J Clin Invest 108(8): 1167-1174, 2001.

17 Evans JM, Donnelly LA, Emslie-Smith AM, Alessi DR and Morris AD: Metformin and reduced risk of cancer in diabetic patients. BMJ 330(7503): 1304-1305, 2005.

18 Ben-Sahra I, Laurent K, Loubat A, Giorgetti-Peraldi S, Colosetti P, Auberger P, Tanti J-F, Le Marchand-Brustel Y and Bost F: The antidiabetic drug metformin exerts an antitumoral effect in vitro and in vivo through a decrease of cyclin D1 level. Oncogene 27(25): 3576-3586, 2008.

19 Bolster DR, Crozier SJ, Kimball SR and Jefferson LS: AMPactivated protein kinase suppresses protein synthesis in rat skeletal muscle through down-regulated mammalian target of rapamycin (MTOR signaling. J Biol Chem 277(27): $23977-$ 23980, 2002.

20 Long YC and Zierath JR: AMP-activated protein kinase signaling in metabolic regulation. J Clin Invest 116(7): 1776-1783, 2006.

21 Hardie DG: Minireview: The AMP-activated protein kinase cascade: The key sensor of cellular energy status. Endocrinology 144(12): 5179-5183, 2003

22 Haring A, Murtola TJ, Talala K, Taari K, Tammela TL and Auvinen A: Antidiabetic drug use and prostate cancer risk in the Finnish randomized study of screening for prostate cancer. Scand J Urol 1-11, 2017.

23 Rothermundt C, Hayoz S, Templeton AJ, Winterhalder R, Strebel RT, Bärtschi D, Pollak M, Lui L, Endt K and Schiess R: Metformin in chemotherapy-naive castration-resistant prostate cancer: A multicenter phase 2 trial (sakk 08/09). Eur Urol 66(3): 468-474, 2014

24 Mesdjian E, Ciesielski L, Valli M, Bruguerolle B, Jadot G, Bouyard P and Mandel P: Sodium valproate: Kinetic profile and effects on GABA levels in various brain areas of the rat. Prog Neuro-Psychopharmacol Biol Psychiatry 6(3): 223-233, 1982.
25 Bradbury C, Khanim F, Hayden R, Bunce C, White D, Drayson M, Craddock C and Turner B: Histone deacetylases in acute myeloid leukaemia show a distinctive pattern of expression that changes selectively in response to deacetylase inhibitors. Leukemia 19(10): 1751-1759, 2005.

26 Ververis K, Rodd AL, Tang MM, El-Osta A and Karagiannis TC: Histone deacetylase inhibitors augment doxorubicin-induced DNA damage in cardiomyocytes. Cell Mol Life Sci 68(24): 4101-4114, 2011.

27 Rosato RR, Almenara JA and Grant S: The histone deacetylase inhibitor MS-275 promotes differentiation or apoptosis in human leukemia cells through a process regulated by generation of reactive oxygen species and induction of p21CIP1/WAF1. Cancer Res 63(13): 3637-3645, 2003.

28 Savickiene J, Borutinskaite V-V, Treigyte G, Magnusson K-E and Navakauskiene R: The novel histone deacetylase inhibitor BML-210 exerts growth inhibitory, proapoptotic and differentiation stimulating effects on the human leukemia cell lines. Eur J Pharmacol 549(1): 9-18, 2006.

29 Martirosyan A, Leonard S, Shi X, Griffith B, Gannett P and Strobl J: Actions of a histone deacetylase inhibitor nsc3852 (5-nitroso-8-quinolinol) link reactive oxygen species to cell differentiation and apoptosis in MCF-7 human mammary tumor cells. J Pharmacol Exp Ther 317(2): 546-552, 2006.

30 Chen Y, Pan RL, Zhang XL, Shao JZ, Xiang LX, Dong XJ and Zhang GR: Induction of hepatic differentiation of mouse bone marrow stromal stem cells by the histone deacetylase inhibitor VPA. J Cell Mol Med 13(8b): 2582-2592, 2009.

31 Sharma S, Symanowski J, Wong B, Dino P, Manno P and Vogelzang N: A phase II clinical trial of oral valproic acid in patients with castration-resistant prostate cancers using an intensive biomarker sampling strategy. Transl Oncol 1(3): 141$147,2008$.

32 Bertolini F, Sukhatme VP and Bouche G: Drug repurposing in oncology patient and health systems opportunities. Nature Reviews Clinical Oncology 12(12): 732-742, 2015.

33 Salpeter SR, Greyber E, Pasternak GA and Salpeter EE: Risk of fatal and nonfatal lactic acidosis with metformin use in type 2 diabetes mellitus. Cochrane Database Syst Rev 4: CD002967, 2010.

34 Tran LN, Kichenadasse G, Butler LM, Centenera MM, Morel KL, Ormsby RJ, Michael MZ, Lower KM and Sykes PJ: The combination of metformin and valproic acid induces synergistic apoptosis in the presence of p53 and androgen signaling in prostate cancer. Mol Cancer Ther 16(12): 26892700, 2017.

35 Zhang X, Zhang X, Huang T, Geng J, Liu M and Zheng J: Combination of metformin and valproic acid synergistically induces cell cycle arrest and apoptosis in clear cell renal cell carcinoma. Int J Clin Exp Pathol 8(3): 2823-2828, 2015.

36 Xia Q, Sung J, Chowdhury W, Chen C-1, Höti N, Shabbeer S, Carducci $\mathrm{M}$ and Rodriguez R: Chronic administration of valproic acid inhibits prostate cancer cell growth in vitro and in vivo. Cancer Res 66(14): 7237-7244, 2006.

37 Tomayko MM and Reynolds CP: Determination of subcutaneous tumor size in athymic (nude) mice. Cancer Chemother Pharmacol 24(3): 148-154, 1989.

38 Sharma VK, Nautiyal V, Goel KK and Sharma A: Assessment of thermal stability of metformin hydrochloride. Asian J Chem 22: 3561-3566, 2010. 
39 Chen S-m, Mukoyama T, Sato N, Yamagata S-I, Arai Y, Satoh N and Ueda S: Induction of nephrotoxic serum nephritis in inbred mice and suppressive effect of colchicine on the development of this nephritis. Pharmacol Res 45(4): 319-324, 2002.

40 Mendler MH, Kanel G and Govindarajan S: Proposal for a histological scoring and grading system for non-alcoholic fatty liver disease. Liver International 25(2): 294-304, 2005.

41 Tran L, Kichenadasse G and Sykes P: Combination therapies using metformin and/or valproic acid in prostate cancer: Possible mechanistic interactions. Curr Cancer Drug Targets 18: 1-14, 2018.

42 Food and Drug Administration: Guidance for industry estimating the maximum safe starting dose in initial clinical trials for therapeutics in adult healthy volunteers. Pharmacol Toxicol: 7-14, 2005.

43 Reagan-Shaw S, Nihal M and Ahmad N: Dose translation from animal to human studies revisited. The FASEB Journal 22(3): 659-661, 2008.

44 Burke J and Thenot J: Determination of antiepileptic drugs. J Chromatogr B Biomed Sci Appl 340: 199-241, 1985.

45 Davoodpour P, Landström $\mathrm{M}$ and Welsh $\mathrm{M}$ : Reduced tumor growth in vivo and increased c-abl activity in PC3 prostate cancer cells overexpressing the shb adapter protein. BMC Cancer 7(1): 161, 2007.
46 Gustavsson H, Tešan T, Jennbacken K, Kuno K, Damber J-E and Welén K: ADAMTS1 alters blood vessel morphology and tsp1 levels in $\mathrm{LNCaP}$ and $\mathrm{LNCaP}-19$ prostate tumors. BMC Cancer 10(1): 288-298, 2010.

47 Shabbeer S, SQ Kortenhorst M, Kachhap S, Galloway N, Rodriguez R and Carducci MA: Multiple molecular pathways explain the anti-proliferative effect of valproic acid on prostate cancer cells in vitro and in vivo. The Prostate 67(10): 1099-1110, 2007.

48 Tai S, Sun Y, Squires JM, Zhang H, Oh WK, Liang CZ and Huang J: PC3 is a cell line characteristic of prostatic small cell carcinoma. The Prostate 71(15): 1668-1679, 2011.

49 Feldman BJ and Feldman D: The development of androgenindependent prostate cancer. Nat Rev Cancer 1(1): 34-45, 2001.

Received October 16, 2018

Revised November 21, 2018

Accepted November 23, 2018 